\title{
Covering the Plane with Translates of a Triangle
}

\author{
Janusz Januszewski
}

Received: 20 December 2007 / Revised: 22 May 2009 / Accepted: 10 June 2009 /

Published online: 30 June 2009

(C) Springer Science+Business Media, LLC 2009

\begin{abstract}
The minimum density of a covering of the plane with translates of a triangle is $\frac{3}{2}$.
\end{abstract}

Keywords Covering · Covering density · Triangle

\section{Introduction}

A collection $\mathcal{C}=\left\{C_{1}, C_{2}, \ldots\right\}$ of planar convex bodies is called a covering of a domain $D \subseteq R^{2}$ provided $\bigcup_{i} C_{i} \supseteq D$. The area of a convex body $C$ is denoted by $|C|$.

For any pair of independent vectors $\mathbf{v}_{1}, \mathbf{v}_{2}$ in the plain, the lattice generated by $\mathbf{v}_{1}$ and $\mathbf{v}_{2}$ is the set of vectors $\left\{k \mathbf{v}_{1}+l \mathbf{v}_{2}: k, l\right.$ integers $\}$. A covering of $R^{2}$ is a lattice covering if it is of the form $(C+\mathbf{v})_{\mathbf{v} \in L}$, where $L$ is a lattice.

The density of a collection $\mathcal{C}$ relative to a bounded domain $D$ is defined as

$$
d(\mathcal{C}, D)=\frac{1}{|D|} \sum_{C \in \mathcal{C}}|C \cap D| .
$$

If the whole plane $R^{2}$ is covered with $\mathcal{C}$ then the lower density of $\mathcal{C}$ in $R^{2}$ is defined as

$$
d_{-}\left(\mathcal{C}, R^{2}\right)=\liminf _{r \rightarrow \infty} d\left(\mathcal{C}, B^{2}(r)\right)
$$

where $B^{2}(r)$ denotes the circle of radius $r$ centered at the origin.

The lattice covering density $\vartheta_{L}(C)$ is defined as the infimum of $d_{-}\left(\mathcal{C}, R^{2}\right)$ taken over all lattice coverings with congruent copies of $C$. The translative covering density

J. Januszewski $(\bowtie)$

Institute of Mathematics and Physics, University of Technology and Life Sciences, ul. Kaliskiego 7, 85-796 Bydgoszcz, Poland

e-mail: januszew@utp.edu.pl 
$\vartheta_{T}(C)$ is defined as the infimum of $d_{-}\left(\mathcal{C}, R^{2}\right)$ taken over all translative coverings with congruent copies of $C$. These infima are attained.

By Fáry's theorem [4], the triangles are the least economical convex sets for a lattice covering; we have $\vartheta_{L}(C) \leq \frac{3}{2}$ for every convex body $C$ and equality holds if and only if $C$ is a triangle (see also [1]).

Since $\vartheta_{T}(C) \leq \vartheta_{L}(C)$, it follows that $\vartheta_{T}(C)<\frac{3}{2}$ for any convex body $C$ in the plane other than a triangle. The aim of this paper is to show that the triangles are the least economical sets for translative covering, i.e. that $\vartheta_{T}(\triangle)=\frac{3}{2}$ for any triangle $\triangle$ (see Problem 2, Chap. 1.3 of [3]).

Various results concerning coverings with convex bodies are discussed in $[2,3,5]$, and [6].

Theorem The minimum density of a covering of the plane with translates of a triangle is $\frac{3}{2}$.

\section{Parts Used for the Covering}

Let $T_{0}$ be the right isosceles triangle whose vertices are $(0,0)$ (called the right vertex), $(-1,0)$, and $(0,1)$ (called the left and the upper vertex, respectively). For any translate $T_{i}$ of $T_{0}$ each of the right, the left, and the upper vertex of $T_{i}$ is defined as the vertex corresponding to the identically named vertex of $T_{0}$. The coordinates of the right vertex of $T_{i}$ are denoted by $\left(x\left(T_{i}\right), y\left(T_{i}\right)\right)$.

Consider a covering $\mathcal{T}$ of the plane with translates of $T_{0}$. We assume that in the covering $\mathcal{T}$ no two triangles coincide. Moreover, we assume that the number of triangles that intersect $B^{2}(r)$ is finite, for each $r>0$.

Let $T_{w}$ and $T_{i}$ be two different triangles of $\mathcal{T}$. We say that $T_{w}$ cuts $T_{i}$ provided at least one of the following three conditions is fulfilled:

(c $\left.c_{1}\right)$ the right vertex of $T_{w}$ belongs to the interior of $T_{i}$;

(c $\left.c_{2}\right)$ the vertical leg of $T_{w}$ intersects both a leg of $T_{i}$ and the hypotenuse of $T_{i}$;

(c $c_{3}$ ) the horizontal leg of $T_{w}$ without the left vertex intersects both a leg of $T_{i}$ and the hypotenuse of $T_{i}$.

For instance, $T^{d}(i)$ cuts $T_{l}$, but $T_{l}$ does not cut $T^{d}(i)$ in the left-hand picture in Fig. 5. If the interiors of $T_{w}$ and $T_{i}$ intersect, then either $T_{w}$ cuts $T_{i}$ or $T_{i}$ cuts $T_{w}$.

Let $r$ be an arbitrary number greater than 4. All triangles of $\mathcal{T}$ that intersect $B^{2}(r-4)$ are denoted by $T_{1}, \ldots, T_{s}$ so that if $i<j$, then either $y\left(T_{i}\right)<y\left(T_{j}\right)$ or $y\left(T_{i}\right)=y\left(T_{j}\right)$ and, at the same time, $x\left(T_{i}\right)>x\left(T_{j}\right)$. Furthermore, denote by $T_{s+1}, \ldots, T_{z}$ the remaining triangles that intersect $B^{2}(r)$.

Let $i \in\{1, \ldots, z\}$ and let $S_{i}$ be the union of the triangles that cut $T_{i}$. The part $U_{i} \subset T_{i}$ used for the covering is defined as the closure of $T_{i} \backslash S_{i}$.

Obviously, $U_{i}$ is a polygon with two sides contained in the legs of $T_{i}$ and with the other sides parallel to the legs of $T_{i}$. Moreover, sets $U_{1}, \ldots, U_{z}$ have pairwise disjoint interiors. We show that sets $U_{1}, \ldots, U_{s}$ cover $B^{2}(r-4)$. Let $a_{0} \in$ $B^{2}(r-4)$. Denote by $n_{1}, \ldots, n_{k}$ all integers such that $a_{0} \in T_{n_{i}}$ for $i=1, \ldots, k$. Obviously, $n_{1}, \ldots, n_{k} \in\{1, \ldots, s\}$. Since $a_{0} \in T_{n_{1}} \cap \cdots \cap T_{n_{k}}$, it follows that there exists an integer $j \in\{1, \ldots, k\}$ such that $a_{0} \in U_{n_{j}}$. Consequently, $B^{2}(r-4) \subset U_{1} \cup \cdots \cup U_{s}$. 

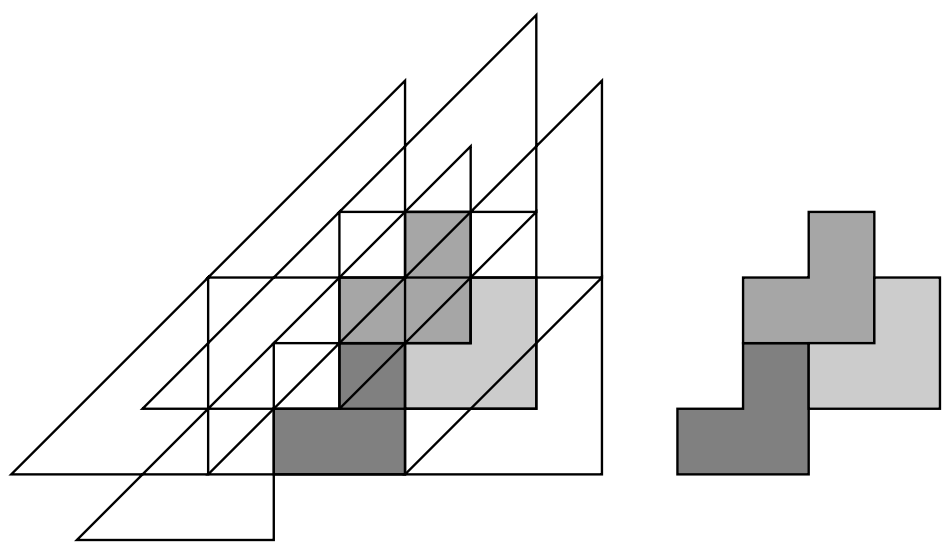

Fig. 1 The optimal lattice covering
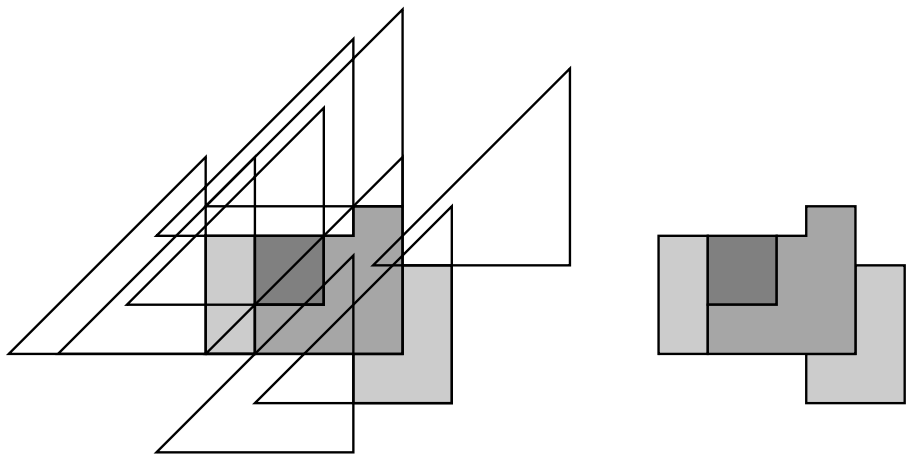

Fig. 2 A translative covering

We say that $U_{i}$ is $n$-tier, if $U_{i}$ has $2 n+2$ sides. Figure 1 illustrates the optimal lattice covering. In the covering $U_{i}$ is 2-tier and $\left|U_{i}\right|=\frac{2}{3}\left|T_{i}\right|$ for each triangle $T_{i}$. Figure 2 illustrates a translative covering.

\section{Types of Triangles}

Note that

$$
\left|U_{i}\right| \leq \frac{1}{3}=\frac{2}{3}\left|T_{i}\right|
$$

provided $U_{i}$ is either 1- or 2-tier. If $U_{i}$ is 1-tier, then $\left|U_{i}\right| \leq \frac{1}{2}\left|T_{i}\right|$. If $U_{i}$ is 2-tier, then $\left|U_{i}\right|=t(1-t)+s(1-t-s)$, where the numbers $t$ and $s$ denote the distances shown in Fig. 3. This 2 nd degree polynomial in two variables reaches its maximum at $s=t=\frac{1}{3}$. Consequently, $\left|U_{i}\right| \leq \frac{1}{3}$. 
Fig. 3 1- and 2-tier parts
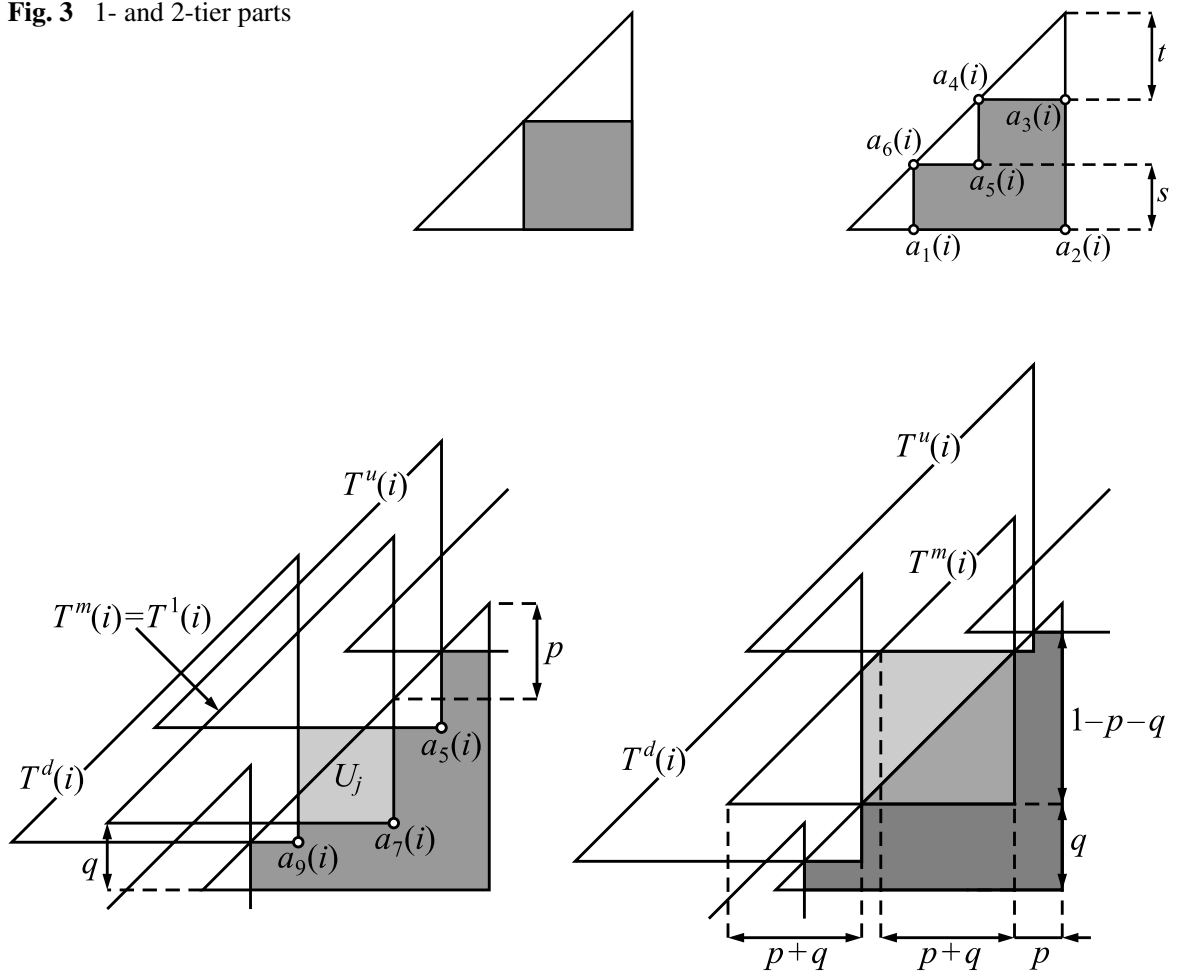

Fig. 4 Triangles of the second type

If $\left|U_{i}\right| \leq \frac{2}{3}\left|T_{i}\right|$ would hold all triangles, it would directly follow that the covering density is at least $\frac{3}{2}$. Unfortunately, if $U_{i}$ is $n$-tier and $n \geq 3$, generally it is not the case here, $\left|U_{i}\right|$ might be arbitrarily close to $\left|T_{i}\right|$. Thus, we have to partition the triangles into small groups of $1,2,3$ or 4 triangles, such that for them

$$
\sum\left|U_{i}\right| \leq \sum \frac{2}{3}\left|T_{i}\right|
$$

holds.

The vertices of $U_{i}$ are denoted by $a_{1}(i), \ldots, a_{2 n+2}(i)$ as presented in Fig. 3.

If $U_{i}$ is $n$-tier, where $n \geq 3$, then we will define triangle $T^{1}(i)$ and, if needed, $T^{2}(i)$ and $T^{3}(i)$. Denote by $T^{u}(i)$ the triangle, the right vertex of which is in $a_{5}(i)$ and by $T^{m}(i)$ the triangle, the right vertex of which is in $a_{7}(i)$. Furthermore, denote by $T^{d}(i)$ the triangle, the right vertex of which is in $a_{9}(i)$ provided $n \geq 4$ (see Fig. 4).

Assume that $n=3$. If $T^{u}(i)$ cuts $T^{m}(i)$, then $T^{1}(i)=T^{m}(i)$. Otherwise, $T^{1}(i)=$ $T^{u}(i)$. We do not define $T^{2}(i)$ (see Fig. 7, where $T^{1}(i)=T_{j}$ ).

Assume that $n \geq 4$. If $T^{u}(i)$ cuts $T^{m}(i)$ and if $T^{d}(i)$ cuts $T^{m}(i)$, then we take $T^{1}(i)=T^{m}(i)$ and we do not define $T^{2}(i)$ (see Fig. 4). Otherwise, we define $T^{1}(i)$ and $T^{2}(i)$ as follows: 

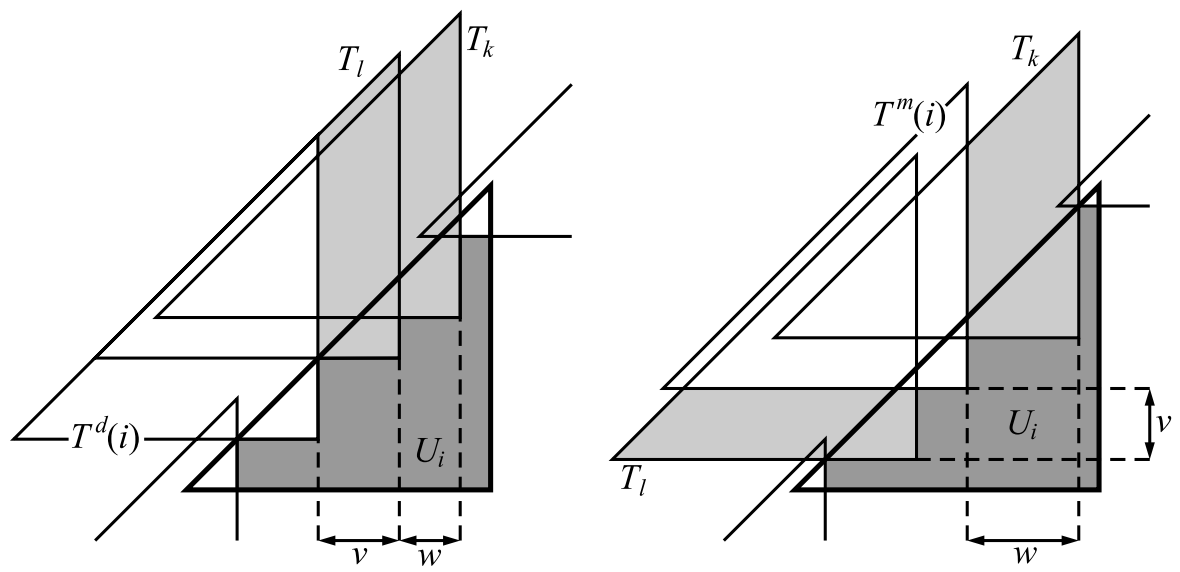

Fig. 5 Triangles of the third type

If $T^{u}(i)$ cuts $T^{m}(i)$, then $T^{1}(i)=T^{m}(i)$.

If $T^{m}(i)$ cuts $T^{u}(i)$, then $T^{1}(i)=T^{u}(i)$.

If $T^{d}(i)$ cuts $T^{m}(i)$, then $T^{2}(i)=T^{m}(i)$.

If $T^{m}(i)$ cuts $T^{d}(i)$, then $T^{2}(i)=T^{d}(i)$ (see Fig. 5 and the left-hand picture in Fig. 6, where $T^{1}(i)=T_{k}$ and $\left.T^{2}(i)=T_{l}\right)$.

The triangles $T_{1}, \ldots, T_{s}$ will be divided into five types. Some of the triangles from among $T_{s+1}, \ldots, T_{z}$ will have defined a type, too. The definition of types is inductive.

First assume that $i=1$.

( $\left.\mathrm{t}_{1}\right)$ If $\left|U_{i}\right| \leq \frac{1}{3}$, then $T_{i}$ is of the first type and $T_{i}$ is basic.

( $\left.\mathrm{t}_{2}\right)$ If $\left|U_{i}\right|>\frac{1}{3}$, if $U_{i}$ is $n$-tier, where $n \geq 4$, and if $T^{2}(i)$ is not defined, then $T_{i}$ is basic and both $T_{i}$ and $T^{1}(i)$ are of the second type.

( $\left.\mathrm{t}_{3}\right)$ If $\left|U_{i}\right|>\frac{1}{3}$, if $U_{i}$ is $n$-tier, where $n \geq 4$, and if $T^{2}(i)$ has been defined, then $T_{i}$ is basic and $T_{i}, T^{1}(i)$ and $T^{2}(i)$ are of the third type.

( $\left.\mathrm{t}_{4}\right)$ If $\left|U_{i}\right|>\frac{1}{3}$, if $U_{i}$ is 3-tier and if $\left|U_{i}\right|+\left|U_{j}\right| \leq \frac{2}{3}$, where $T_{j}=T^{1}(i)$, then $T_{i}$ is basic and both $T_{i}$ and $T^{1}(i)$ are of the fourth type.

(t5) If $\left|U_{i}\right|>\frac{1}{3}$, if $U_{i}$ is 3-tier and if $\left|U_{i}\right|+\left|U_{j}\right|>\frac{2}{3}$, where $T_{j}=T^{1}(i)$, then $T_{i}$ is basic and $T_{i}, T^{1}(i), T^{1}(j)$ and $T^{2}(j)$ (provided it is defined) are of the fifth type. $T^{1}(j)$ is denoted by $T^{2}(i)$ and $T^{2}(j)$ (provided it is defined) is denoted by $T^{3}(i)$.

Now assume that $i \in\{2, \ldots, s\}$ is the smallest integer such that the type of $T_{i}$ has not been yet defined. The type of $T_{i}$ is defined by conditions $\left(\mathrm{t}_{1}\right)-\left(\mathrm{t}_{5}\right)$.

Assume that $T_{i}$ and $T_{l}$, where $i \neq l$, are basic triangles and that $T^{\lambda}(i)$ and $T^{\mu}(l)$ have been defined, where $\lambda, \mu \in\{1,2,3\}$. Let $j$ be the integer such that $T_{j}=T^{\lambda}(i)$. Since there is only one integer $\eta \neq j$ and there is only one integer $\kappa$ such that $a_{2}(j)=$ $a_{\kappa}(\eta)$, it follows that $T^{\lambda}(i) \neq T^{\mu}(l)$. 

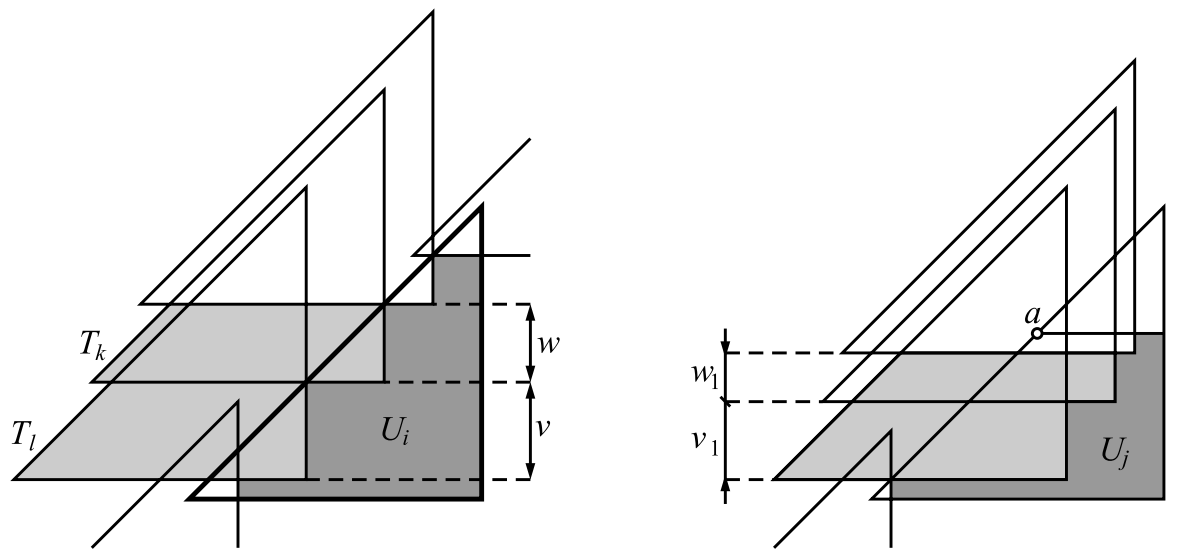

Fig. 6 Triangles of the third and the fifth type

\section{Size of Parts Used for the Covering}

Obviously, if $T_{i}$ is a basic triangle of the first type, then $\left|U_{i}\right| \leq \frac{2}{3}\left|T_{i}\right|$.

Let $T_{i}$ be a basic triangle of the second type. Denote by $j$ the integer such that $T_{j}=T^{1}(i)$. Observe that

$$
\left|U_{j} \backslash T_{i}\right| \leq \frac{1}{6}
$$

Denote by $p$ the distance between the vertical leg of $T_{i}$ and the vertical leg of $T^{1}(i)$ (see Fig. 4). Furthermore, denote by $q$ the distance between the horizontal leg of $T^{1}(i)$ and the horizontal leg of $T_{i}$. If $p+q \geq \frac{1}{2}$, then $\left|U_{j} \backslash T_{i}\right| \leq \frac{1}{4}\left|T_{j}\right|=\frac{1}{8}$ (see the left-hand picture in Fig. 4). If $p+q<\frac{1}{2}$, then

$$
\left|U_{j} \backslash T_{i}\right| \leq \frac{1}{2}-\frac{1}{2}(1-p-q)^{2}-(p+q)^{2}
$$

(see the right-hand picture in Fig. 4, where $U_{j} \backslash T_{i}$ is contained in the light-grey trapezoid). Since the maximum of this upper bound is reached when $p+q=\frac{1}{3}$, it follows that $\left|U_{j} \backslash T_{i}\right| \leq \frac{1}{6}$. This implies that

$$
\left|U_{i}\right|+\left|U_{j}\right| \leq \frac{1}{2}+\frac{1}{6}=\frac{2}{3}=\frac{2}{3}\left(\left|T_{i}\right|+\left|T_{j}\right|\right) .
$$

Let $T_{i}$ be a basic triangle of the third type (see Fig. 5 and the left-hand picture in Fig. 6).

If $T^{1}(i)=T^{u}(i)$, then denote by $w$ the distance between the vertical leg of $T^{u}(i)$ and the vertical leg of $T^{m}(i)$. If $T^{1}(i)=T^{m}(i)$, then denote by $w$ the distance between the horizontal leg of $T^{u}(i)$ and the horizontal leg of $T^{m}(i)$. If $T^{2}(i)=T^{m}(i)$, then denote by $v$ the distance between the vertical leg of $T^{m}(i)$ and the vertical leg of $T^{d}(i)$. If $T^{2}(i)=T^{d}(i)$, then denote by $v$ the distance between the horizontal leg 

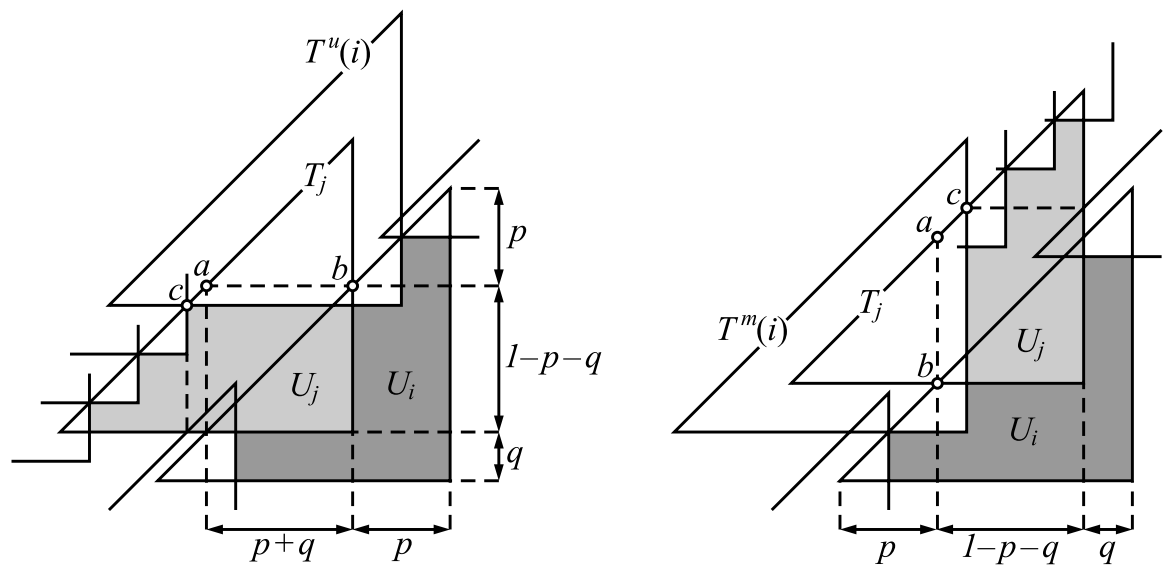

Fig. 7 Triangles of the fourth type

of $T^{m}(i)$ and the horizontal leg of $T^{d}(i)$. Furthermore, denote by $k$ and $l$ the integers such that $T_{k}=T^{1}(i)$ and $T_{l}=T^{2}(i)$. It is easy to see that

$$
\begin{aligned}
\left|U_{i}\right|+\left|U_{k}\right|+\left|U_{l}\right| & \leq \frac{1}{2}+v(1-v)+w(1-w) \\
& \leq \frac{1}{2}+\frac{1}{4}+\frac{1}{4}=1=\frac{2}{3}\left(\left|T_{i}\right|+\left|T_{k}\right|+\left|T_{l}\right|\right) .
\end{aligned}
$$

If $T_{i}$ is a basic triangle of the fourth type and if $T^{1}(i)=T_{j}$, then

$$
\left|U_{i}\right|+\left|U_{j}\right| \leq \frac{2}{3}\left(\left|T_{i}\right|+\left|T_{j}\right|\right)
$$

Let $T_{i}$ be a basic triangle of the fifth type and let $T^{1}(i)=T_{j}$. Obviously, $\left|U_{i}\right|>\frac{1}{3}$ and $\left|U_{i}\right|+\left|U_{j}\right|>\frac{2}{3}$.

If $T_{j}=T^{m}(i)$, then denote by $p$ the distance between the vertical leg of $T_{j}$ and the vertical leg of $T_{i}$ and denote by $q$ the distance between the horizontal leg of $T_{j}$ and the horizontal leg of $T_{i}$ (see the left-hand picture in Fig. 7). If $T_{j}=T^{u}(i)$, then denote by $p$ the distance between the horizontal leg of $T_{j}$ and the horizontal leg of $T_{i}$ and denote by $q$ the distance between the vertical leg of $T_{j}$ and the vertical leg of $T_{i}$ (see the right-hand picture in Fig. 7).

Note that $\frac{1}{2}<p+q<0.64$. It is easy to see that

$$
\left|U_{i}\right| \leq q(1-q)+p(1-q-p)+\frac{1}{4} p^{2} .
$$

If $p+q \leq \frac{1}{2}$, then, by a simple calculus argument, this upper bound does not exceed $\frac{1}{3}$. Consequently, $T_{i}$ is not a basic triangle of the fifth type. Moreover,

$$
\left|U_{i}\right|+\left|U_{j}\right| \leq q(1-q)+p(1-p-q)+\frac{1}{4} p^{2}+\frac{1}{2}-\frac{1}{2}(p+q)^{2} .
$$


If $p+q \geq 0.64$, then, by a simple calculus argument, this upper bound does not exceed $\frac{2}{3}$. Consequently, $T_{i}$ is not a basic triangle of the fifth type.

We show that $U_{j}$ is $n$-tier, where $n \geq 4$. Assume the opposite, that $n \leq 3$.

If $T_{j}=T^{m}(i)$, then denote by $b$ the common point of the hypotenuse of $T_{i}$ and the vertical leg of $T_{j}$, by $a$ the common point of the hypotenuse of $T_{j}$ and the horizontal straight line containing $b$, and denote by $c$ the common point of the hypotenuse of $T_{j}$ and the horizontal leg of $T^{u}(i)$ (see the left-hand picture in Fig. 7). It is easy to see that the area of the part of $U_{i}$ lying above the horizontal straight line containing $b$ does not exceed $\frac{1}{2} \cdot \frac{1}{2} p^{2}$. Since $U_{j}$ is 1-, 2- or 3-tier, the area of the part of $U_{j}$ lying on the left side of the vertical straight line containing $c$ does not exceed $\frac{2}{3} \cdot \frac{1}{2}(1-p-q)^{2}$.

If $T_{j}=T^{u}(i)$, then denote by $b$ the common point of the hypotenuse of $T_{i}$ and the horizontal leg of $T_{j}$, by $a$ the common point of the hypotenuse of $T_{j}$ and the vertical straight line containing $b$, and denote by $c$ the common point of the hypotenuse of $T_{j}$ and the vertical leg of $T^{m}(i)$ (see the right-hand picture in Fig. 7). The area of the part of $U_{i}$ lying on the left side of the vertical straight line containing $b$ does not exceed $\frac{1}{2} \cdot \frac{1}{2} p^{2}$. The area of the part of $U_{j}$ lying above the horizontal straight line containing $c$ does not exceed $\frac{2}{3} \cdot \frac{1}{2}(1-p-q)^{2}$.

Since $p+q>\frac{1}{2}$, it follows that

$$
\begin{aligned}
\left|U_{i}\right|+\left|U_{j}\right| \leq & \frac{1}{3}(1-p-q)^{2}+\frac{1}{4} p^{2}+q(1-q) \\
& +p(1-p-q)+(p+q)(1-p-q) .
\end{aligned}
$$

This 2nd degree polynomial in two variables reaches its maximum at $p=\frac{1}{3}$ and $q=\frac{1}{6}$, and therefore $\left|U_{i}\right|+\left|U_{j}\right| \leq \frac{2}{3}$, which is a contradiction.

Since $U_{j}$ is $n$-tier, where $n \geq 4$, there are two possibilities: either $T^{2}(j)$ is defined or $T^{2}(j)$ is not defined.

Assume that $T^{2}(j)$ is not defined (see Fig. 8; $T_{j}=T^{m}(i)$ in the left-hand picture and $T_{j}=T^{u}(i)$ in the right-hand picture). Denote by $k$ the integer such that $T_{k}=$ $T^{1}(j)$. The area of $U_{k} \backslash T_{j}$ is not greater than $\frac{1}{6}$ (see (1)). Consequently,

$$
\left|U_{i}\right|+\left|U_{j}\right|+\left|U_{k}\right| \leq q(1-q)+p(1-p-q)+\frac{1}{4} p^{2}+\frac{1}{2}-\frac{1}{2}(p+q)^{2}+\frac{1}{6} .
$$

This 2nd degree polynomial in two variables reaches its maximum at $p=\frac{2}{7}$ and $q=\frac{1}{7}$. Consequently,

$$
\left|U_{i}\right|+\left|U_{j}\right|+\left|U_{k}\right| \leq \frac{37}{42}<1=\frac{2}{3}\left(\left|T_{i}\right|+\left|T_{j}\right|+\left|T_{k}\right|\right) .
$$

Now assume that $T^{2}(j)$ is defined. Denote by $k$ and $l$ the integers such that $T^{1}(j)=T_{k}$ and $T^{2}(j)=T_{l}$.

Assume that $T_{j}=T^{m}(i)$. Denote by $R_{j}$ the part of $T_{j}$ lying under the horizontal straight line containing $a$.

If $T_{l}=T^{d}(j)$, then take $v_{2}=0$ and denote by $v_{1}$ the distance between the horizontal leg of $T_{m}(i)$ and the horizontal leg of $T_{l}$ (see Fig. 10 and the right-hand picture in Fig. 6). 


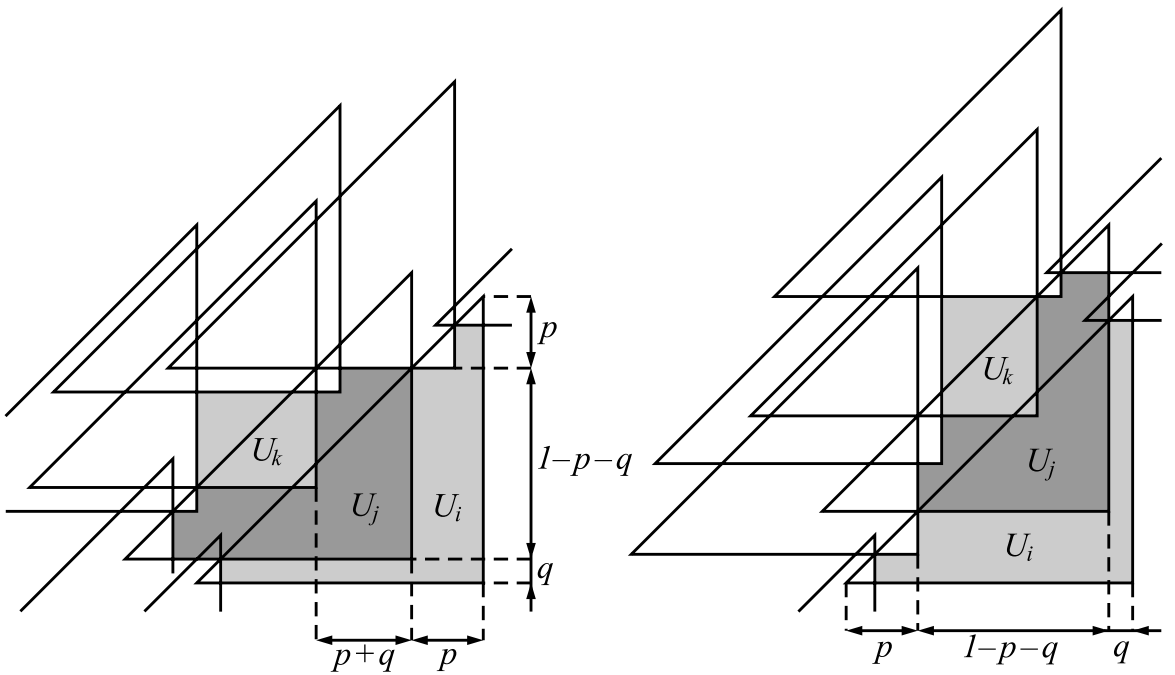

Fig. 8 Triangles of the fifth type, $T^{2}(j)$ is not defined
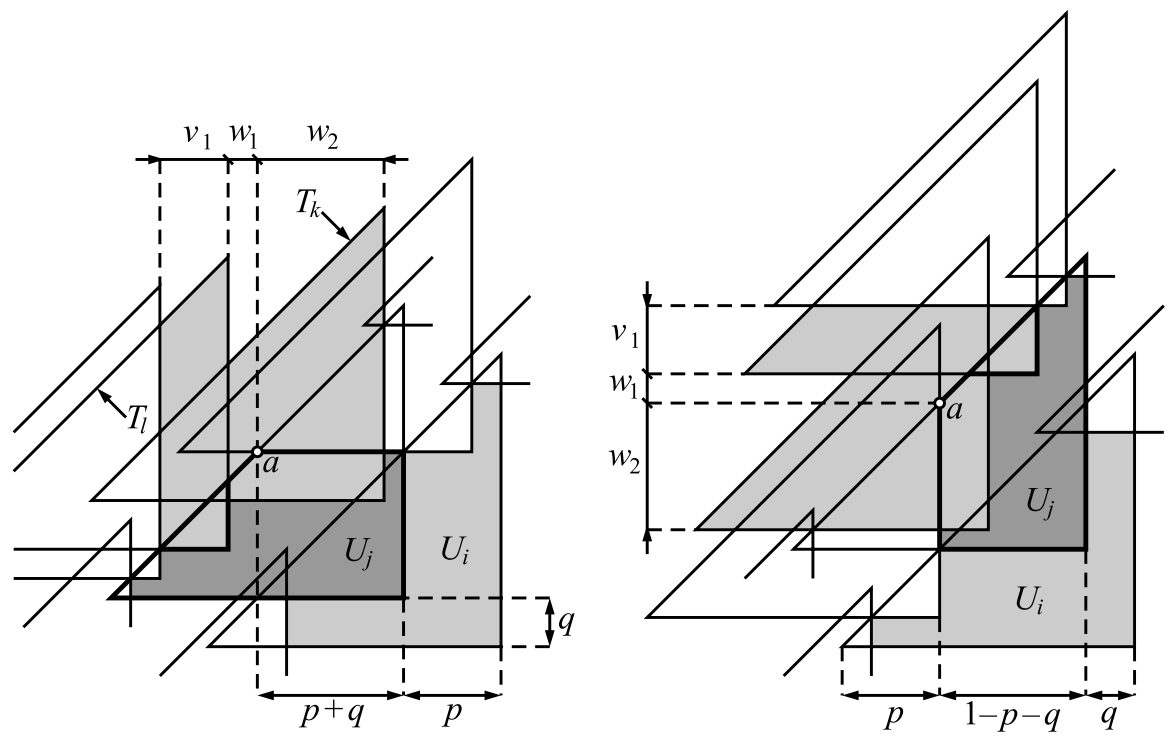

Fig. 9 Triangles of the fifth type, $T^{2}(j)$ is defined

If $T_{l}=T^{m}(j)$ and if $a \in T_{k} \backslash T_{l}$, then take $v_{2}=0$ and denote by $v_{1}$ the distance between the vertical leg of $T_{l}$ and the vertical leg of $T^{d}(j)$ (see the left-hand picture in Fig. 9).

If $T_{l}=T^{m}(j)$ and if $a \in T^{d}(j)$, then take $v_{1}=0$ and denote by $v_{2}$ the distance between the vertical leg of $T_{l}$ and the vertical leg of $T^{d}(j)$ (see the right-hand picture in Fig. 11). 

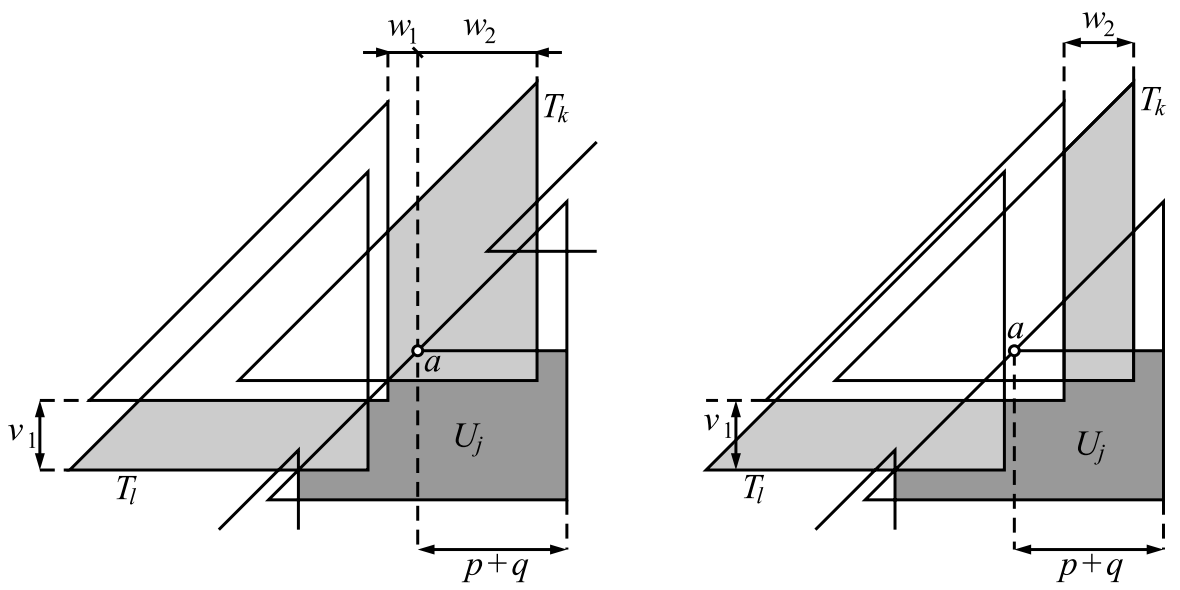

Fig. 10 Triangles of the fifth type, $T^{2}(j)=T^{d}(j)$
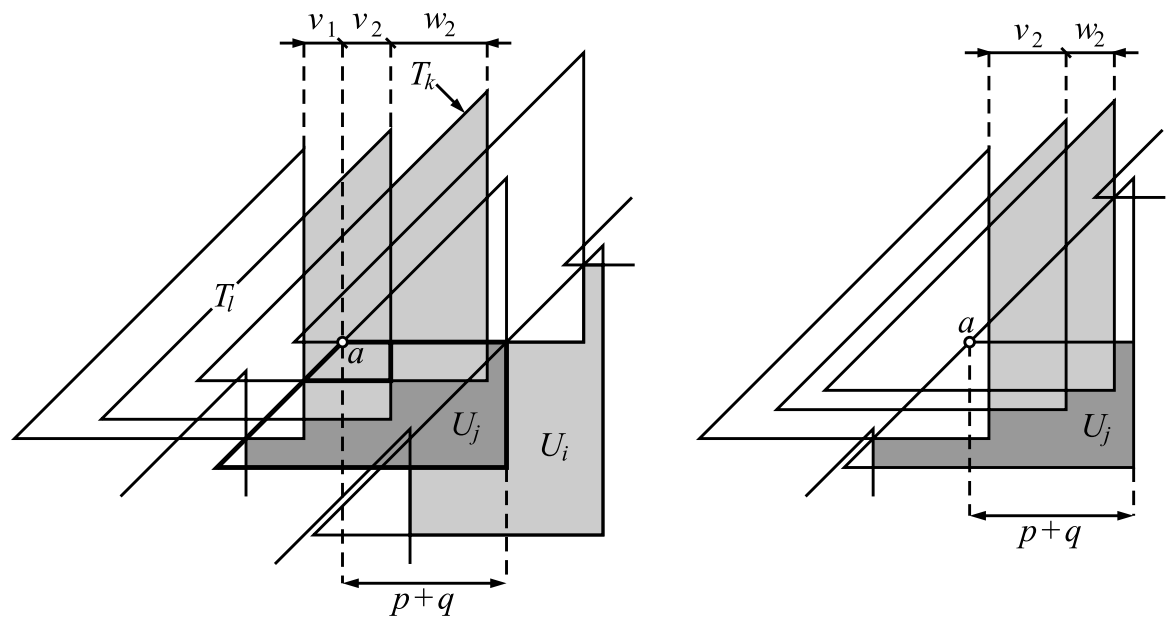

Fig. 11 Triangles of the fifth type, $T^{1}(j)=T^{u}(j)$

If $T_{l}=T^{m}(j)$ and if $a \in T_{l} \backslash T^{d}(j)$, then denote by $v_{1}$ the distance between $a$ and the vertical leg of $T^{d}(j)$ and denote by $v_{2}$ the distance between $a$ and the vertical leg of $T_{l}$ (see the left-hand picture in Fig. 11).

If $T_{k}=T^{m}(j)$, then take $w_{2}=0$ and denote by $w_{1}$ the distance between the horizontal leg of $T^{u}(i)$ and the horizontal leg of $T_{k}$ (see the right-hand picture in Fig. 6).

If $T_{k}=T^{u}(j)$ and if $a \notin T_{k} \backslash T^{m}(j)$, then take $w_{1}=0$ and denote by $w_{2}$ the distance between the vertical leg of $T_{m}(i)$ and the vertical leg of $T_{k}$ (see Fig. 11).

If $T_{k}=T^{u}(j)$ and if $a \in T_{k} \backslash T^{m}(j)$, then denote by $w_{1}$ the distance between $a$ and the vertical leg of $T^{m}(i)$ and by $w_{2}$ the distance between $a$ and the vertical leg of $T_{k}$ (see the left-hand pictures in Figs. 9 and 10).

Consider the case when $v_{2}=0$ (e.g. see the left-hand picture in Fig. 9). 
Observe that $\frac{1}{2} v_{1}^{2} \leq 0.05$. If $\frac{1}{2} v_{1}^{2}>0,05$, then

$$
\left|U_{i}\right|+\left|U_{j}\right|<q(1-q)+p(1-p-q)+\frac{1}{4} p^{2}+\frac{1}{2}-\frac{1}{2}(p+q)^{2}-0.05 .
$$

This 2nd degree polynomial in two variables reaches its maximum at $p=\frac{2}{7}$ and $q=\frac{1}{7}$. Consequently, $\left|U_{i}\right|+\left|U_{j}\right|<\frac{2}{3}$, which is a contradiction. This implies that $v \leq \sqrt{0.1}<0.32$ and $\left|U_{l} \backslash R_{j}\right| \leq v_{1}\left(1-v_{1}\right)<0.32(1-0.32)<0.22$.

Obviously,

$$
\left|U_{k} \backslash R_{j}\right| \leq\left(w_{1}+w_{2}\right)\left(1-w_{1}-w_{2}\right)+\frac{1}{2} w_{2}^{2}=w_{1}-w_{1}^{2}+w_{2}-\frac{1}{2} w_{2}^{2}-2 w_{1} w_{2} .
$$

By a simple calculus argument, this upper bound does not exceed $p+q-\frac{1}{2}(p+q)^{2}$ provided $0 \leq w_{1} \leq 1-p-q$ and $0 \leq w_{2} \leq p+q$.

Hence, the sum $\left|U_{i}\right|+\left|U_{j}\right|+\left|U_{k}\right|+\left|U_{l}\right|$ is smaller than

$$
q(1-q)+p(1-p-q)+\frac{1}{4} p^{2}+\frac{1}{2}-\frac{1}{2}(p+q)^{2}+p+q-\frac{1}{2}(p+q)^{2}+0.22 .
$$

This 2nd degree polynomial in two variables reaches its maximum at $p=\frac{2}{5}$ and $q=\frac{1}{5}$. Consequently,

$$
\left|U_{i}\right|+\left|U_{j}\right|+\left|U_{k}\right|+\left|U_{l}\right|<\frac{4}{3}=\frac{2}{3}\left(\left|T_{i}\right|+\left|T_{j}\right|+\left|T_{k}\right|+\left|T_{l}\right|\right) .
$$

Consider the case when $v_{2} \neq 0$ (see Fig. 11).

Observe that $\frac{1}{2} v_{1}^{2}+v_{1} v_{2} \leq 0.05$, otherwise, by (2), $\left|U_{i}\right|+\left|U_{j}\right|<\frac{2}{3}$, which is a contradiction.

It is easy to see that

$$
\left|\left(U_{k} \cup U_{l}\right) \backslash R_{j}\right| \leq v_{1}\left(1-v_{1}-v_{2}\right)+v_{2}+w_{2}-\frac{1}{2}\left(v_{2}+w_{2}\right)^{2}+v_{2}\left(w_{2}-v_{1}\right) .
$$

Since $v_{2}+w_{2} \leq p+q<0.64$, it follows that

$$
\left|\left(U_{k} \cup U_{l}\right) \backslash R_{j}\right|<v_{1}\left(1-v_{1}-v_{2}\right)+p+q-\frac{1}{2}(p+q)^{2}+v_{2}\left(0.64-v_{2}-v_{1}\right) .
$$

If $v_{1} \geq 0,0 \leq v_{2} \leq 1$ and if $\frac{1}{2} v_{1}^{2}+v_{1} v_{2} \leq 0.05$, then, by a calculus argument,

$$
v_{1}\left(1-v_{1}-v_{2}\right)+v_{2}\left(0.64-v_{2}-v_{1}\right)<0.22 \text {. }
$$

Consequently, the sum $\left|U_{i}\right|+\left|U_{j}\right|+\left|U_{k}\right|+\left|U_{l}\right|$ does not exceed

$q(1-q)+p(1-p-q)+\frac{1}{4} p^{2}+\frac{1}{2}-\frac{1}{2}(p+q)^{2}+p+q-\frac{1}{2}(p+q)^{2}+0.22<\frac{4}{3}$.

If $T_{j}=T^{u}(i)$, then, arguing in a similar way (e.g. compare the left-hand and the right-hand picture in Fig. 9), we also obtain

$$
\left|U_{i}\right|+\left|U_{j}\right|+\left|U_{k}\right|+\left|U_{l}\right| \leq \frac{2}{3}\left(\left|T_{i}\right|+\left|T_{j}\right|+\left|T_{k}\right|+\left|T_{l}\right|\right) .
$$




\section{The Proof}

Proof Since the problem is invariant under affine transformations of the plane, we can consider coverings with translates of the right isosceles triangle $T_{0}$.

Obviously, $\vartheta_{T}\left(T_{0}\right) \leq \vartheta_{L}\left(T_{0}\right)=\frac{3}{2}$. We show that $\vartheta_{T}\left(T_{0}\right) \geq \frac{3}{2}$.

Let $\mathcal{T}$ be an arbitrary covering of the plane with translates of $T_{0}$. We can assume that no triangles in $\mathcal{T}$ coincide (we are looking for the minimum covering density).

Let $r$ be an arbitrary number greater than 4 . We can assume that only finitely many of the triangles in $\mathcal{T}$ intersect $B^{2}(r)$, otherwise the lower density of $\mathcal{T}$ would be infinite. We define parts used for the covering and types of triangles as in Sects. 2 and 3 .

Denote by $A_{h}$ the set of integers $i$ such that $T_{i}$ is of the $h$ th type for $h=1, \ldots, 5$ and let $A=A_{1} \cup \cdots \cup A_{5}$. From the consideration presented in Sect. 4 we have

$$
\sum_{i \in A}\left|T_{i}\right| \geq \frac{3}{2} \sum_{i \in A}\left|U_{i}\right|
$$

Obviously, $T_{i} \subset B^{2}(r-4+\sqrt{2})$ for $i \in\{1, \ldots, s\}$. Moreover, each triangle for which a type has been defined is contained in $B^{2}(r-2+\sqrt{2})$.

Since

$$
B^{2}(r) \supset \bigcup_{i \in A} T_{i} \supset \bigcup_{i \in A} U_{i} \supset B^{2}(r-4),
$$

it follows that

$$
\sum_{i=1}^{z}\left|T_{i} \cap B^{2}(r)\right| \geq \sum_{i \in A}\left|T_{i}\right| \geq \frac{3}{2} \sum_{i \in A}\left|U_{i}\right| \geq \frac{3}{2} \pi(r-4)^{2} .
$$

Consequently,

$$
\liminf _{r \rightarrow \infty} d\left(\mathcal{T}, B^{2}(r)\right) \geq \frac{3}{2} \lim _{r \rightarrow \infty} \frac{(r-4)^{2}}{r^{2}}=\frac{3}{2} .
$$

Acknowledgement The author would like to thank the anonymous referee for discovering a mistake in the first version of the paper.

\section{References}

1. Besicovitch, A.S.: Measure of asymmetry of convex curves. J. Lond. Math. Soc. 23, 237-240 (1948)

2. Böröczky, K. Jr.: Finite Packing and Covering. Cambridge Tracts in Mathematics, vol. 154. Cambridge University Press, Cambridge (2004)

3. Brass, P., Moser, W., Pach, J.: Research Problems in Discrete Geometry. Springer, New York (2005)

4. Fáry, I.: Sur la densité des réseaux de domaines convexes. Bull. Soc. Math. Fr. 178, 152-161 (1950)

5. Fejes Tóth, G., Kuperberg, W.: Packing and covering with convex sets. In: Gruber, P.M., Wills, J.M. (eds.) Handbook of Convex Geometry, vol. A, B, pp. 799-860. North-Holland, Amsterdam (1993)

6. Fejes Tóth, G.: Packing and covering. In: Goodman, J.E., O’Rourke, J. (eds.) Handbook of Discrete and Computational Geometry. Discrete Mathematics and its Applications, vol. 1, pp. 25-52. Chapman Hall/CRC, Boca Raton (2004) 